- 研究论文・

\title{
人参中农药多残留的超高效液相色谱-串联质谱分析方法研究
}

\author{
陈丽娜 ${ }^{a, b}$ 宋凤瑞 ${ }^{a}$ 郑 重 ${ }^{a}$ 邢俊鹏 ${ }^{a}$ 刘志强*, $a$ 刘淑莹 ${ }^{a}$ \\ $\left({ }^{a}\right.$ 中国科学院长春应用化学研究所 长春质谱中心 长春 130022) \\ ( ${ }^{b}$ 中国科学院研究生院 北京 100049)
}

\begin{abstract}
摘要 以传统中药人参为研究对象, 建立一种同时检测人参中 46 种农药多残留的基质固相分散-超高效液相色谱-串 联质谱(MSPD-UPLC-MS/MS)方法. 前处理方法分别采用 $N$-丙基乙二胺(PSA)和乙腈作为 MSPD 的分散剂和洗脱剂, 检 测方法采用正负离子同时扫描，利用超高效液相色谱-串联质谱在分时段多反应监测模式下进行定量和定性分析. 大 部分农药在 5 500 $\mu \mathrm{g} / \mathrm{kg}$ 范围内线性关系良好, 在低 $(10 \mu \mathrm{g} / \mathrm{kg})$ 、中 $(50 \mu \mathrm{g} / \mathrm{kg})$ 、高 $(100 \mu \mathrm{g} / \mathrm{kg})$ 三个浓度水平上的添加回 收率平均值在 $70 \% \sim 110 \%$ 之间，相对标准偏差(RSD)小于 $15 \%(n=3)$, 定量限小于 $0.01 \mathrm{mg} / \mathrm{kg}$, 能够满足农药多残留分 析要求. 该方法通用性强、选择性好、灵敏度高、简单廉价.
\end{abstract}

关键词 基质固相分散法; 超高效液相色谱-串联质谱; 人参; 多农药残留

\section{Studies on the Determination Method of Pesticide Multi-residues in Ginseng by Ultra Performance Liquid Chromatography Tandem Mass Spectrometry}

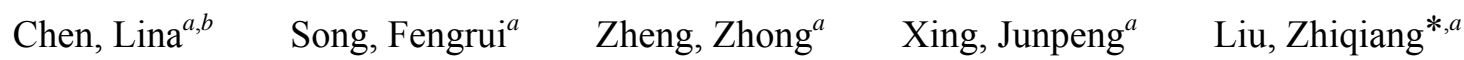 \\ Liu, Shuying ${ }^{a}$ \\ ( ${ }^{a}$ Changchun Institute of Applied Chemistry Chinese Academy of Sciences, Changchun Centre of Mass Spectrometry, \\ Changchun 130022) \\ ( ${ }^{b}$ Graduate School of Chinese Academy of Sciences, Beijing 100049)
}

\begin{abstract}
Taking the traditional herbal, ginseng, as the object in the paper, a comprehensive analytical method based on matrix solid-phase dispersion-ultra performance liquid chromatography tandem mass spectrometry (MSPD-UPLC-MS/MS) has been developed for the simultaneous determination of 46 pesticide residues in Ginseng. The sample was prepared by MSPD. Primary secondary amine (PSA) was used as dispersant, and acetonitrile was used as eluant. Both positive and negative ionization modes were used in the detection. Qualitative and quantitative analysis was carried out by UPLC-MS/MS under multiple reaction monitoring mode (MRM). The linear range of this method was $5 \sim 500.0 \mu \mathrm{g} / \mathrm{kg}$. The limits of quantification (LOQ) for the 46 pesticides were less than $0.01 \mathrm{mg} / \mathrm{kg}$. The mean recoveries at the three spiked levels of $10 \sim 100 \mu \mathrm{g} / \mathrm{kg}$ were $70 \% \sim 110 \%$, with relative standard deviations (RSD) better than $15 \%(n=3)$. The method is robust, selective, sensitive, rapid and cheap.
\end{abstract}

Keywords matrix solid-phase dispersion; ultra performance liquid chromatography tandem mass spectrometry; ginseng; multi-residue pesticides

\footnotetext{
*E-mail: liuzq@ciac.j1.cn

Received October 26, 2011; revised November 30, 2011; accepted January 5, 2012.

Project supported by the National Key Technology R\&D Program in the 11th Five year Plan of China (No. 2009ZX09308-006) and Innovation Method Fund of China (No. 2010IM030400).

“十一五”国家科技支撑计划(No. 2009ZX09308-006)和国家科技部创新方法工作专项(No. 2010IM030400)资助项目.
} 
人参是我国的珍贵中药材, 也是中药材出口的重要 品种. 目前商场上流通的人参, 绝大部分来源于人工种 植, 因此, 无论在人参的生长期, 还是在收割后, 都不 可避免的需要使用农药, 所以, 人参的农药残留十分值 得关注. 特别是近些年, 各国对中药材质量的重视, 农 药残留的检测项目不断增加, 最大残留限量大幅度降 低, 限量标准也日趋严格. 如何在有限的资源配置下节 约人力、物力, 提高工作效率, 是农药残留检测人员面 临的问题, 由此多农药残留检测技术应运而生.

目前主要的多残留检测手段有气相色谱技术 ${ }^{[1]}$ 、气 相色谱一质谱技术 ${ }^{[2,3]}$ 、液相色谱-串联质谱技术 ${ }^{[4-5]}$ 以及 气相色谱-串联质谱技术 ${ }^{[6]}$, 然而, 随着有机氯类农药的 禁用以及大极性易降解农药的不断产生, 液相色谱一串 联质谱技术的应用越来越普及 ${ }^{[7]}$. 本文将超高效液相色 谱与串联质谱技术联用，进一步减少分析时间，提高了 工作效率.

从样品前处理方法上讲, 目前的多农药残留分析方 法普遍存在着诸如操作麻烦、过程复杂、净化效果不够 理想、适用农药种类或基质种类有限等缺陷. 因此, 人 参中农残的相关报道大部分只针对特定的某种或几种 农药 ${ }^{[8 \sim 10]} .1995$ 年, Fillion 等 ${ }^{[1]}$ 应用 SPE-GC-SIM-MS 和 GC-FPD 分析了人参中 110 种农药, 2010 年, Douglas 等 ${ }^{[12]}$ 应用 SPE-GPC 方法净化, GC-qMS-SIM 和 GC-HRTOFMS 检测方法分析了人参中 170 种农药, 此两种方 法虽然实现了多残留分析, 但是仍存在共同的缺点, 就 是前处理过程繁琐, 有机溶剂使用量大, 不仅实验费用 昂贵, 更容易污染环境, 因此, 建立一种快速, 简单, 廉 价, 环保的农药多残留前处理方法就更为有意义. 基质 固相分散(MSPD)技术是在固相萃取基础上发展起来的 一种可同时进行提取和分离的样品前处理技术 ${ }^{[13,14]}$. 它 适合多种类农药的多残留分析, 而且样品处理速度快, 溶剂与样品使用量少.

本研究探讨了利用 MSPD 提取净化、UPLC-MS/MS 分离检测中药人参中 46 种农药多残留量的可能性, 建 立了 MSPD-UPLC-MS/MS 检测人参中农药多残留量的 分析方法. 并将此方法应用于实际样品的分析, 获得较 好的分析结果.

\section{1 实验部分}

\section{1 仪器与试剂}

UPLC-MS/MS (ACQUITY UPLC, Xevo TM TQ, Waters 公司), 配有 ESI 离子源, 三重四级杆质量分析 器; Acquity UPLC BEH C18 色谱柱 $(1.7 \mu \mathrm{m}, 2.1 \times 100$ mm, Waters 公司); BEH C18 保护柱 $(1.7 \mu \mathrm{m}, 2.1 \times 5 \mathrm{~mm}$, Waters 公司); 研针(玛瑙材质).

乙腈、甲醇(色谱纯, Fisher，美国); 乙酸乙酯、丙酮 (分析纯, 北京试剂), 甲酸、乙酸(色谱纯, 天津科密欧公 司); PSA, C18 与石墨化碳粉末(购于 Sigma 公司); 弗洛 里硅土(分析纯, 阿拉丁)、薄层层析硅胶(化学纯, 青岛 海洋化工)和柱层析中性氧化铝(AR, 上海路都化学试 剂), 高温活化后, 加质量分数 $5 \%$ 的超纯水灭活, 用前 $130{ }^{\circ} \mathrm{C}$ 烘 $4 \mathrm{~h}$; Milli-Q 纯净水(美国 Millipore 公司); 农药 标准物质: 纯度 $>95 \%$ (分别来自 Sigma 公司和西域公 司).

\section{2 色谱条件}

Waters ACQUITY UPLC BEH C18 柱 $(1.7 \mu \mathrm{m}$, $2.1 \times 100 \mathrm{~mm}$ ); 流动相: 乙腈 $(\mathrm{A})$ 和水溶液 $($ 含 $0.1 \%$ 甲 酸)(B), 梯度洗脱程序: $0 \sim 1.0 \min , 10 \% \sim 30 \% \mathrm{~A} ; 1.0 \sim$ $9.0 \mathrm{~min}, 30 \% \sim 90 \% \mathrm{~A} ; 9.0 \sim 10.0 \mathrm{~min}, 90 \% \sim 90 \% \mathrm{~A}$; $10.0 \sim 11.0 \mathrm{~min}, 90 \% \sim 70 \% \mathrm{~A} ; 11.0 \sim 13.0 \mathrm{~min}, 70 \% \sim$ $10 \% \mathrm{~A} ; 13.0 \sim 15.0 \mathrm{~min}, 10 \% \sim 10 \%$ A. 流速 $0.3 \mathrm{~mL} / \mathrm{min}$; 柱温 $35{ }^{\circ} \mathrm{C}$; 样品室温度 $10{ }^{\circ} \mathrm{C}$; 进样量 $7.5 \mu \mathrm{L}$.

\section{3 质谱条件}

电喷雾离子源;正、负离子扫描; 毛细管电压 $3.2 \mathrm{kV}$; 离子源温度 $150{ }^{\circ} \mathrm{C}$; 去溶剂气温度 $400{ }^{\circ} \mathrm{C}$; 去溶剂气流 量 $700 \mathrm{~L} / \mathrm{h}$; 锥孔气流量 $50 \mathrm{~L} / \mathrm{h}$; 碰撞气体为氩气, 流速 $0.16 \mathrm{~L} / \mathrm{h}$; 多反应监测 (MRM)模式检测, 46 种农药的质 谱分析参数见表 1 .

\section{4 标准储备液和工作液的制备}

准确称取适量的各农药标准品, 用甲醇溶解并定容 至 $10 \mathrm{~mL}$, 配制成 $1000 \mathrm{mg} / \mathrm{L}$ 标准储备液, 于 $-18{ }^{\circ} \mathrm{C}$ 下 保存. 准确量取各农药标准储备液 $50 \mu \mathrm{L}$, 用甲醇定容 至 $50 \mathrm{~mL}$, 配制成 $1.0 \mathrm{mg} / \mathrm{L}$ 混合标准储备液. 使用时, 用甲醇逐级稀释成混合标准工作液, 于 $4{ }^{\circ} \mathrm{C}$ 下保存.

\section{5 样品的处理}

将待检测的人参饮片粉碎后, 过 9 号篮, 得到人参 样品粉末. 准确称取 $1 \mathrm{~g}$ 人参样品粉末于研钵中, 加入 $400 \mathrm{mg}$ PSA 分散剂, 充分研磨成均匀粉末; 在层析柱下 端放入小块脱脂棉, 加入研磨好的混合体, 轻轻敲实, 最后在混合物上方覆盖一层脱脂棉，并用玻璃棒压实; 用 $4 \mathrm{~mL}$ 乙腈淋洗层析柱, 并用柱芯将淋洗液全部压出, 收集淋洗液于试管中, 用氮气于 $30{ }^{\circ} \mathrm{C}$ 下吹至近干, 用 $V$ (甲醇) $: V$ (水) $=3: 2$ 定容至 $1 \mathrm{~mL}$, 过 $0.2 \mu \mathrm{m}$ 滤膜, 供 UPLC-MS/MS 检测. 
表 146 种农药的质谱分析参数 ${ }^{a}$

Table 1 MS parameters for 46 pesticides

\begin{tabular}{|c|c|c|c|c|c|}
\hline No. & Compd. & Retention/min & Mass transitions $(\mathrm{m} / \mathrm{z})$ & Cone voltage/V & Collision energy/eV \\
\hline \multirow{2}{*}{1} & \multirow{2}{*}{ 3-羟基克百威(Carbofuran-3-hydroxy) } & \multirow{2}{*}{2.410} & $238.1 / 163.1$ & \multirow{2}{*}{19} & 14 \\
\hline & & & $238.1 / 181.1$ & & 10 \\
\hline \multirow{2}{*}{2} & \multirow{2}{*}{ 倍硫磷砜(Fenthion-sulfone) } & \multirow{2}{*}{4.935} & 311.1/125.1, & \multirow{2}{*}{29} & 20 \\
\hline & & & $311.1 / 279.0$ & & 17 \\
\hline \multirow{2}{*}{3} & \multirow{2}{*}{ 倍硫磷亚砜(Fenthion-sulfoxide) } & \multirow{2}{*}{3.930} & $295.2 / 280.0$ & \multirow{2}{*}{29} & 18 \\
\hline & & & $295.2 / 109.1$ & & 32 \\
\hline \multirow{2}{*}{4} & \multirow{2}{*}{ 苯醚甲环唑(Difenoconazole) } & \multirow{2}{*}{7.195} & $406.1 / 251.0$ & \multirow{2}{*}{31} & 24 \\
\hline & & & $406.1 / 337.0$ & & 17 \\
\hline \multirow{2}{*}{5} & \multirow{2}{*}{ 吡虫啉(Imidacloprid) } & \multirow{2}{*}{2.515} & $256.4 / 175.1$ & \multirow{2}{*}{19} & 17 \\
\hline & & & $256.4 / 209.1$ & & 16 \\
\hline \multirow{2}{*}{6} & \multirow{2}{*}{ 吡蚜酮(Pymetrozine) } & \multirow{2}{*}{1.895} & $218.3 / 105.1$ & 24 & 18 \\
\hline & & & $218.3 / 88.1$ & 24 & 20 \\
\hline 7 & 莍入咸 $(\mathrm{D}$ & 3010 & $210.2 / 168.1$ & 12 & 7 \\
\hline 1 & 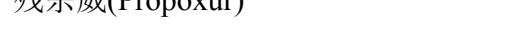 & 3.910 & $210.2 / 111.1$ & 13 & 15 \\
\hline 8 & 中酰明(Tohfonozido) & 6750 & $353.3 / 297.1$ & 10 & 7 \\
\hline$\delta$ & 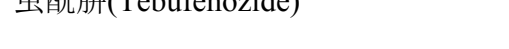 & 0.150 & $353.3 / 133.1$ & 7 & 20 \\
\hline 0 & 敌䊩Dromoni) & 5225 & $218.1 / 162.0$ & 28 & 15 \\
\hline 9 & 故杵(PropaniI) & 5.250 & $218.1 / 127.1$ & 15 & 27 \\
\hline 10 & 敉㺃暥 & 6165 & $272.1 / 129.1$ & 21 & 16 \\
\hline 10 & 故早肗(Napropamide) & 0.165 & $272.1 / 171.1$ & 16 & 18 \\
\hline 11 & 丁草㫨(Putoblor) & 8655 & $312.4 / 162.1$ & 20 & 21 \\
\hline 11 & J早抆(Dutactior) & 0.053 & $312.4 / 238.2$ & 21 & 11 \\
\hline 12 & 它苜磷 (D- & 7270 & $374.2 / 222.1$ & 22 & 21 \\
\hline 12 & 企困桝多(Pyrazopnos) & 1.210 & $374.2 / 346.0$ & 33 & 15 \\
\hline 13 & 啶中胖(Acetaminrid) & 2625 & $223.1 / 126.1$ & 20 & 20 \\
\hline 10 & 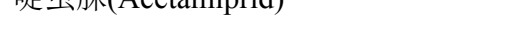 & $2.02 \mathrm{~J}$ & $223.1 / 56.1$ & 20 & 16 \\
\hline 14 & 对咇菌环㫨 $(C$ vprodinil) & 7125 & $226.2 / 93.1$ & 41 & 30 \\
\hline 14 & 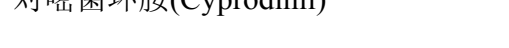 & 1.120 & $226.2 / 108.2$ & 41 & 23 \\
\hline 15 & 䒠索磷(Fonsulfothion) & 4685 & $308.9 / 252.8$ & 25 & 18 \\
\hline 15 & 手系侈(Fensulfotnion) & 4.685 & $308.9 / 280.9$ & 25 & 15 \\
\hline 16 & 田莫䁙(Alachlor) & 6530 & $270.0 / 238.1$ & 10 & 12 \\
\hline 10 & 中华脑(Alacnior) & 0.550 & $270.0 / 162.0$ & 19 & 21 \\
\hline 17 & 田芙威(Corhorv1) & 4215 & $202.2 / 145.1$ & 16 & 10 \\
\hline 11 & T示敒(Calva1yi) & $4.54 J$ & 202.2/127.1 & 10 & 27 \\
\hline 10 & 田雪寻 & 4565 & $280.2 / 220.2$ & 20 & 13 \\
\hline 18 & 中租灭(Metalaxyl) & 4.565 & $280.2 / 248.1$ & 20 & 9 \\
\hline 19 & 睛菌唑(Mvclobutanil) & 5820 & $289.1 / 70.1$ & 25 & 16 \\
\hline & & 0.020 & $289.1 / 125.1$ & & 34 \\
\hline 20 & 抗枒威(Pirimicarh) & 4575 & $239.1 / 72.1$ & 25 & 20 \\
\hline 20 & 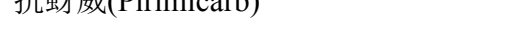 & $4.5 / 5$ & $239.1 / 182.2$ & 25 & 17 \\
\hline 21 & 古百威 $(C)$ & 4280 & $222.2 / 165.2$ & 17 & 12 \\
\hline 21 & 兄日㑈(Carboiuran) & 4.280 & $222.2 / 123.1$ & 17 & 20 \\
\hline 22 & 喹禾灵(Ouizalofon-etbyl) & 7940 & $373.1 / 299.1$ & 30 & 18 \\
\hline 22 & 性木炎(Vuizaloiop-etinyi) & 1.940 & $373.1 / 91.0$ & 30 & 30 \\
\hline 23 & 磷㫨(Phosnhamidon) & 2210 & $300.0 / 127.0$ & 21 & 21 \\
\hline 20 & 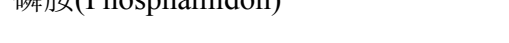 & 5.210 & $300.0 / 174.1$ & 21 & 13 \\
\hline
\end{tabular}




\begin{tabular}{|c|c|c|c|c|c|}
\hline No. & Compd. & Retain time/min & Mass transitions $(\mathrm{m} / \mathrm{z})$ & Cone voltage $/ \mathrm{V}$ & Collision energy/eV \\
\hline \multirow{2}{*}{24} & \multirow{2}{*}{ 氯唑啉(Isazofos) } & \multirow{2}{*}{9.310} & $314.0 / 161.9$ & \multirow{2}{*}{23} & 16 \\
\hline & & & $314.0 / 271.9$ & & 13 \\
\hline \multirow{2}{*}{25} & \multirow{2}{*}{ 咯菌腈(Fludioxonil) ${ }^{b}$} & \multirow{2}{*}{5.595} & $247.0 / 126.1$ & \multirow{2}{*}{37} & 29 \\
\hline & & & 247.0/180.1 & & 27 \\
\hline \multirow{2}{*}{26} & \multirow{2}{*}{ 马拉氧磷(Malaoxon) } & \multirow{2}{*}{3.800} & $315.1 / 127.1$ & \multirow{2}{*}{17} & 12 \\
\hline & & & $315.1 / 99.1$ & & 25 \\
\hline \multirow{2}{*}{27} & \multirow{2}{*}{ 猛杀威(Promecarb) } & \multirow{2}{*}{5.820} & $208.1 / 151.1$ & \multirow{2}{*}{17} & 8 \\
\hline & & & 208.1/109.1 & & 19 \\
\hline \multirow{2}{*}{28} & \multirow{2}{*}{ 嘧啶磷(Pirimiphos-ethyl) } & \multirow{2}{*}{8.850} & $334.2 / 198.2$ & \multirow{2}{*}{29} & 21 \\
\hline & & & $334.2 / 182.1$ & & 24 \\
\hline \multirow{2}{*}{29} & \multirow{2}{*}{ 嘧菌酯(Azoxystrobin) } & \multirow{2}{*}{5.685} & $404.1 / 372.0$ & 20 & 13 \\
\hline & & & $404.1 / 343.8$ & & 23 \\
\hline 30 & 灭多威(Methomvl) & 1.955 & $162.9 / 88.1$ & 18 & 10 \\
\hline & & & $162.9 / 106.2$ & 10 & 10 \\
\hline 31 & 灭菌磷(Ditalimfos) & 6.515 & $300.1 / 148.0$ & 19 & 18 \\
\hline & & 0.013 & $300.1 / 244.0$ & 19 & 13 \\
\hline 32 & 灭古磷(Fthonronhos) & 6105 & $243.0 / 173.1$ & 20 & 15 \\
\hline 32 & 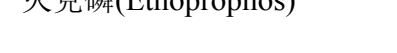 & 0.105 & $243.0 / 130.9$ & 20 & 21 \\
\hline 33 & 㘔湊(Thiamethoxam) & 2090 & $292.1 / 211.0$ & 17 & 13 \\
\hline & & & $292.1 / 131.9$ & & 20 \\
\hline 34 & 㘔嗪酮(Buprofezin) & 9875 & $306.2 / 201.1$ & 19 & 11 \\
\hline 34 & 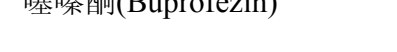 & $9.8 / 3$ & $306.2 / 116.1$ & 19 & 15 \\
\hline 35 & 杀铃胒 (Triflumuron $)^{b}$ & 7020 & $356.9 / 154.0$ & 10 & 13 \\
\hline & & 1.020 & $356.9 / 176.1$ & 19 & 23 \\
\hline 36 & 二唑醇(Triadimenol) & 5.300 & $296.1 / 70.1$ & 13 & 10 \\
\hline & & & $296.1 / 227.1$ & & 10 \\
\hline 37 & 二叻酮(Triadimefon) & 5850 & 294.1/197.1 & 23 & 16 \\
\hline & 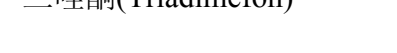 & 0.000 & $294.1 / 69.1$ & $2 J$ & 20 \\
\hline 38 & 速灭威(Metolcarb) & 3.580 & $166.0 / 109.1$ & 14 & 10 \\
\hline 30 & 特谱枞(Diniconarole) & 6685 & $326.2 / 70.1$ & 33 & 25 \\
\hline 39 & 特谱㟇(Diniconazole) & 6.685 & $326.2 / 158.9$ & 33 & 29 \\
\hline 40 & 涕灭威砜(Aldicarb-sulfone) & 1.815 & $240.2 / 223.1$ & 11 & 8 \\
\hline & & & $240.2 / 76.1$ & 11 & 13 \\
\hline 41 & 戊唑醇(Tebuconazole) & 6.240 & $308.2 / 70.1$ & 27 & 19 \\
\hline & & $0.2+0$ & $308.2 / 125.1$ & 27 & 36 \\
\hline 12 & 西玛津(Simazine) & 3520 & $202.1 / 104.1$ & 30 & 26 \\
\hline 42 & 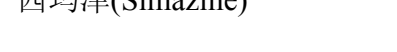 & $3 . J 20$ & $202.1 / 96.1$ & 50 & 23 \\
\hline 43 & 乙嘧硫磷(Etrimfos) & 7.290 & $293.1 / 265.0$ & 29 & 17 \\
\hline & & & 293.1/125.0 & & 23 \\
\hline 44 & 异丙甲草胺(Metolachlor) & 6.520 & $284.1 / 252.1$ & 18 & 15 \\
\hline & & 0.020 & $284.1 / 176.2$ & 10 & 24 \\
\hline 45 & 异丙威(Isonrocarb) & 4.735 & $211.2 / 194.1$ & 9 & 6 \\
\hline $4 J$ & 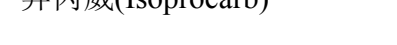 & (4.150 & $211.2 / 95.1$ & 3 & 19 \\
\hline 46 & 仲丁威(Fenobucarb) & 5.400 & $208.2 / 95.1$ & 18 & 13 \\
\hline & & & $208.2 / 152.1$ & 10 & 7 \\
\hline
\end{tabular}

${ }^{a}$ Scan type: ESI+. ${ }^{b}$ Scan type: ESI- 


\section{2 结果和讨论}

\section{1 农药品种的篮选}

首先对中药中农药的使用情况做实际研究, 根据调 研的农药使用实际情况，参考国际国内标准，结合农药 毒性、半衰期、禁限用要求等, 对农药进行初步笁选, 再 对上述篮选出的农药按结构进行分类, 最终篮选出适合 HPLC-MS 检测的 50 种农药，从结构上基本涉及了除有 机氯与拟除虫菊酯类外的各类农药.

\section{2 样品前处理方法的优化}

\section{2 .1 分散剂和洗脱剂的选择}

为了找到最佳的提取条件, 考察了几种分散剂和洗 脱剂的提取效果. 考察的分散剂有硅胶(SI)、中性氧化铝 (AL2O3-N)、弗洛里硅土(Florisil), PSA, PSA十石墨化碳 $(\mathrm{GCB})$. 净化效果依次为 $\mathrm{PSA}+\mathrm{GCB} \geqslant \mathrm{PSA}>\mathrm{AL} 2 \mathrm{O} 3-$ $\mathrm{N}>\mathrm{C} 18>\mathrm{SI}>$ Florisil. 洗脱溶剂考察了农药多残留分 析的常用溶剂, 乙腈、甲醇、乙酸乙酯、丙酮, 净化效 果依次为乙腈 $>$ 乙酸乙酯 $>$ 丙酮 $>$ 甲醇. 并对净化效果 较好的提取方案进行回收率考察, 由于众多文献中报 道, 用一定的酸度洗脱可以提高碱不稳定性农药的回收 率，所以对于 PSA-乙腈组合，还考察了不同酸度对回收 率的影响. 初步尝试 50 种农药, 加标水平为 $20 \mathrm{ng} / \mathrm{g}$, 实 验结果如表 2. PSA 做分散剂, 乙腈或丙酮做洗脱溶剂 时回收率最好, 而 $\mathrm{GCB}$ 的加入会导致低极性农药的回 收率降低, 丙酮做洗脱剂时, 虽然回收率与 PSA 相似, 但净化效果不如乙腈, 同时, 实验结果表明, 酸化乙腈 并没有改善农药的回收率, 相反, 还使部分农药的回收 率降低. 因此, 最终确定乙腈作洗脱溶剂.

\subsection{2 样品与分散剂比例的选择}

分别考察了 $0.5: 0.25 ; 0.5: 0.5 ; 0.5: 1 ; 0.5: 1.5$; $1.0: 0.8 ; 1.0: 0.6 ; 1.0: 0.4 ; 1.0: 0.2$, 实验结果如图 1 , 表明物料比为 $1: 0.4$ 时, 净化效果与回收率都比较好.

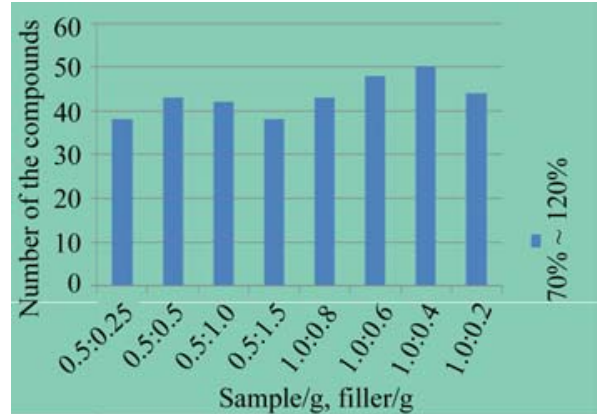

图 1 样品与分散剂的比例

Figure 1 Ratio of sample to filler

\subsection{3 洗脱溶剂体积的选择}

洗脱溶剂的体积直接影响目标分析物能否被洗脱 下来, 本实验考察了随着乙腈体积的增加提取效率的变 化情况, 实验结果如图 2. 当洗脱溶剂体积从 $4 \mathrm{~mL}$ 到 6 $\mathrm{mL}$ 时, 回收率无明显变化, 因此本实验洗脱溶剂的体 积选择 $4 \mathrm{~mL}$.

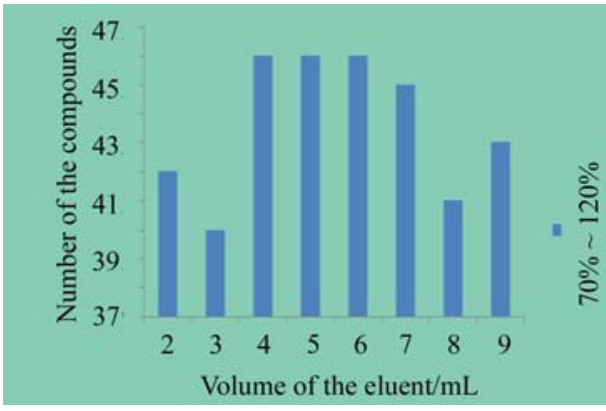

图 2 洗脱溶剂的体积

Figure 2 Volume of the eluent

\section{3 色谱条件的优化}

选择 BEH C18 柱 $(1.7 \mu \mathrm{m}, 2.1 \mathrm{~mm} \times 100 \mathrm{~mm})$ 作为分 析柱，比较了乙腈一水和甲醇-水两个体系的分离效果. 与乙腈-水体系相比，甲醇一水体系使部分农药的保留时

表 2 不同萃取填料和洗脱剂对回收率的影响

Table 2 Influence on the recovery with different fillers and eluents

\begin{tabular}{|c|c|c|c|c|c|c|}
\hline \multirow{2}{*}{ Fillers solvents } & \multicolumn{3}{|c|}{$\mathrm{PSA}+\mathrm{GCB}$} & \multicolumn{3}{|c|}{ PSA } \\
\hline & $\mathrm{MeCN}$ & EtOAc & Acetone & $\mathrm{MeCN}$ & EtOAc & Acetone \\
\hline Number $^{a}$ & 39 & 28 & 36 & 50 & 33 & 50 \\
\hline \multirow{2}{*}{ Fillers solvents } & \multicolumn{3}{|c|}{ Almina-N } & \multicolumn{3}{|c|}{ PSA } \\
\hline & $\mathrm{MeCN}$ & EtOAC & Acetone & $\mathrm{MeCN}$ & $0.1 \%$ Acetone $/ \mathrm{MeCN}$ & $1 \%$ Acetone $/ \mathrm{MeCN}$ \\
\hline Number $^{a}$ & 42 & 39 & 39 & 50 & 37 & 35 \\
\hline
\end{tabular}

${ }^{a}$ Number of compounds whose recoveries in the range of $70 \% \sim 120 \%$. 
间向后延迟，峰形变宽，负离子扫描时的灵敏度明显不 如前者, 因此, 本实验选择乙腈-水体系作为流动相. 为 了进一步改善峰形和灵敏度, 本实验还考察了不同添加 剂(甲酸、乙酸、甲酸铵、乙酸铵)及浓度对流动相的影 响. 由于各农药的化学性质和保留性质相差较大, 选择 梯度洗脱方式, 通过优化流动相梯度洗脱条件实现了 46 种农药的有效分离.

在洗脱过程中, 发现进样溶剂的极性对保留时间较 短的色谱峰存在明显的溶剂效应, 严重影响定量效果, 为此, 本实验也对进样溶剂的组成进行了考察, 并确定 $V$ (甲醇) $: V$ (水) $=3: 2$ 作为最终进样溶剂.

\section{4 质谱条件的优化}

采用初始流动相稀释各个农药标准品, 对每个农药 标准品溶液进行全扫描, 确定每种农药的母离子质荷 比, 然后对母离子进行子离子扫描, 并从中选出两个丰 度比较高的子离子作为定量和定性离子, 建立多离子反 应监测(MRM)离子对. 然后, 在结合流动相的情况下, 对其它质谱参数如干燥气流速、锥孔气流速、毛细管电 压、干燥气温度等进行优化, 确保灵敏度达到最佳值.

\section{5 线性范围和定量限}

用空白样品加入标准溶液制作 7 点系列标准工作 溶液, 在选定的色谱和质谱条件下进行测定, 以各成分 的峰面积对基质标准系列浓度作图来确定其线性关系. 在实验条件下, 以信噪比为 10 时, 定量离子对信号对应 的样品浓度来估算定量限(LOQ). 46 种农药的线性方 程、线性范围、相关系数和定量限见表 3. 毒死蜱、恶 唑禾草灵、二嗪磷和氧皮蝇磷四种农药因线性关系不好, 不做进一步考察. 所以, 对于此 46 种农药, 本方法灵敏 度高, 线性范围宽, 能够满足人参中农药残留分析的要 求.

\section{6 方法回收率和精密度}

用空白人参样品, 分别添加不同浓度的标准溶液 后，浴旋混匀，并放置过夜，再按照样品前处理方法进 行操作，用超高效液相色谱-串联四极杆质谱进样测定， 每个浓度水平重复 3 次, 其回收率和精密度结果见表 4. 大部分人参样品的加标回收率在 $70 \% \sim 110 \%$ 范围内， 相对标准偏差 $<15 \%$, 符合农药多残留分析的要求.

表 346 种农药的线性方程、线性范围、相关系数和定量限

Table 3 Linear equations, linear ranges, correlation coefficients and the quantification limits of 46 pesticides

\begin{tabular}{|c|c|c|c|c|}
\hline Compd. & Linear equation & Linear range $/\left(\mu \mathrm{g} \cdot \mathrm{kg}^{-1}\right)$ & Correlation coefficient (r2) & $(\mathrm{LOQ}) /\left(\mu \mathrm{g} \cdot \mathrm{kg}^{-1}\right)$ \\
\hline 3-羟基克百威(Carbofuran-3-hydroxy) & $y=614 x+2242$ & $5.0 \sim 500$ & 0.997 & 0.2 \\
\hline 倍硫磷砜(Fenthion-sulfone) & $y=99 x-31$ & $5.0 \sim 500$ & 0.995 & 5.0 \\
\hline 倍硫磷亚砜(Fenthion-sulfoxide) & $y=587 x+12513$ & $5.0 \sim 500$ & 0.993 & 0.5 \\
\hline 苯醚甲环唑(Difenoconazole) & $y=1119 x+2200$ & $5.0 \sim 100$ & 0.990 & 0.1 \\
\hline 吡虫啉(Imidacloprid) & $y=148 x+507$ & $5.0 \sim 500$ & 0.999 & 0.1 \\
\hline 吡蚜酮(Pymetrozine) & $y=1668 x-3099$ & $5.0 \sim 500$ & 0.999 & 0.1 \\
\hline 残杀威(Propoxur) & $y=405 x-596$ & $5.0 \sim 500$ & 0.996 & 0.1 \\
\hline 虫酰肼(Tebufenozide) & $y=305 x+6099$ & $5.0 \sim 500$ & 0.993 & 0.1 \\
\hline 敌稗(Propanil) & $y=28 x-82$ & $10.0 \sim 500$ & 0.997 & 10.0 \\
\hline 敌草胺(Napropamide) & $y=2077 x+10992$ & $5.0 \sim 100$ & 0.989 & 0.1 \\
\hline 丁草胺(Butachlor) & $y=249 x+225$ & $5.0 \sim 500$ & 0.999 & 0.2 \\
\hline 定菌磷(Pyrazophos) & $y=1402 x+4130$ & $5.0 \sim 250$ & 0.999 & 0.1 \\
\hline 啶虫脒(Acetamiprid) & $y=1054 x+7696$ & $5.0 \sim 500$ & 0.998 & 0.1 \\
\hline 对嘧菌环胺(Cyprodinil) & $y=1612 x+20700$ & $5.0 \sim 500$ & 0.997 & 0.1 \\
\hline 丰索磷(Fensulfothion) & $y=111 x-270$ & $5.0 \sim 100$ & 0.998 & 2.0 \\
\hline 甲草胺(Alachlor) & $y=99 x+2390$ & $5.0 \sim 500$ & 0.996 & 5.0 \\
\hline 甲萗威(Carbaryl) & $y=686 x+3282$ & $5.0 \sim 500$ & 0.998 & 0.5 \\
\hline 甲霜灵(Metalaxyl) & $y=1760 x+8067$ & $5.0 \sim 500$ & 0.998 & 0.1 \\
\hline 腈菌唑(Myclobutanil) & $y=158 x+770$ & $5.0 \sim 500$ & 0.999 & 0.5 \\
\hline 抗蚜威(Pirimicarb) & $y=6107 x-7296$ & $5.0 \sim 500$ & 0.999 & 0.2 \\
\hline 克百威(Carbofuran) & $y=2311 x+8393$ & $5.0 \sim 500$ & 0.999 & 0.1 \\
\hline 喹禾灵(Quizalofop-ethyl) & $y=56 x+356$ & $5.0 \sim 250$ & 0.990 & 2.0 \\
\hline 磷胺(Phosphamidon) & $y=1317 x+5105$ & $5.0 \sim 500$ & 0.991 & 0.2 \\
\hline
\end{tabular}




\begin{tabular}{|c|c|c|c|c|}
\hline Compd. & Linear equation & Linear range $/\left(\mu \mathrm{g} \cdot \mathrm{kg}^{-1}\right)$ & Correlation coefficient (r2) & $(\mathrm{LOQ}) /\left(\mu \mathrm{g} \cdot \mathrm{kg}^{-1}\right)$ \\
\hline 氯唑啉(Isazofos) & $y=920 x+3208$ & $5.0 \sim 250$ & 0.992 & 0.1 \\
\hline 咯菌腈(Fludioxonil) & $y=700 x+6638$ & $5.0 \sim 250$ & 0.995 & 0.1 \\
\hline 马拉氧磷(Malaoxon) & $y=1292 x+1257$ & $5.0 \sim 500$ & 0.999 & 0.1 \\
\hline 猛杀威(Promecarb) & $y=520 x+334$ & $5.0 \sim 500$ & 0.998 & 0.1 \\
\hline 嘧啶磷(Pirimiphos-ethyl) & $y=4489 x+43677 x$ & $5.0 \sim 500$ & 0.997 & 0.1 \\
\hline 嘧菌酯(Azoxystrobin) & $y=125 x-328$ & $5.0 \sim 100$ & 0.996 & 0.1 \\
\hline 灭多威(Methomyl) & $y=273 x-312$ & $5.0 \sim 500$ & 0.999 & 0.5 \\
\hline 灭菌磷(Ditalimfos) & $y=782 x+4184$ & $5.0 \sim 500$ & 0.999 & 0.2 \\
\hline 灭克磷(Ethoprophos) & $y=527 x+5088$ & $5.0 \sim 500$ & 0.998 & 5.0 \\
\hline 噻虫嗪(Thiamethoxam) & $y=149 x+252$ & $5.0 \sim 500$ & 0.999 & 0.5 \\
\hline 噻嗪酮(Buprofezin) & $y=312 x+875$ & $5.0 \sim 500$ & 0.996 & 0.2 \\
\hline 三唑醇(Triadimenol) & $y=66 x+390$ & $5.0 \sim 400$ & 0.998 & 1.0 \\
\hline 三唑酮(Triadimefon) & $y=152 x+1118$ & $5.0 \sim 500$ & 0.995 & 2.0 \\
\hline 杀铃艮(Triflumuron) & $y=512 x+7866$ & $5.0 \sim 500$ & 0.992 & 0.2 \\
\hline 速灭威(Metolcarb) & $y=220 x+82$ & $5.0 \sim 400$ & 0.995 & 0.2 \\
\hline 特谱唑(Diniconazole) & $y=496 x-871$ & $5.0 \sim 500$ & 0.997 & 1.0 \\
\hline 涕灭威砜(Aldicarb-sulfone) & $y=226 x-1516$ & $5.0 \sim 500$ & 0.998 & 5.0 \\
\hline 戊唑醇(Tebuconazole) & $y=574 x+8797$ & $5.0 \sim 400$ & 0.996 & 0.2 \\
\hline 西玛津(Simazine) & $y=341 x-542$ & $5.0 \sim 500$ & 0.997 & 1.0 \\
\hline 乙嘧硫磷(Etrimfos) & $y=305 x+636$ & $5.0 \sim 500$ & 0.999 & 1.0 \\
\hline 异丙甲草胺(Metolachlor ) & $y=1353 x+18563$ & $5.0 \sim 500$ & 0.997 & 0.1 \\
\hline 异丙威(Isoprocarb) & $y=176 x+2115$ & $5.0 \sim 500$ & 0.997 & 0.5 \\
\hline 仲丁威(Fenobucarb) & $y=649 x+10038$ & $5.0 \sim 500$ & 0.995 & 0.1 \\
\hline
\end{tabular}

表 4 回收率和精密度

Figure 4 Recoveries and RSDs of 46 pesticides in Ginseng

\begin{tabular}{|c|c|c|c|c|c|c|}
\hline \multirow[b]{2}{*}{ Compd. } & \multicolumn{2}{|c|}{ Spiked $10 \mu \mathrm{g} / \mathrm{kg}$} & \multicolumn{2}{|c|}{ Spiked $50 \mu \mathrm{g} / \mathrm{kg}$} & \multicolumn{2}{|c|}{ Spiked $100 \mu \mathrm{g} / \mathrm{kg}$} \\
\hline & Recovery $/ \%$ & $\begin{array}{c}\mathrm{RSD} / \% \\
(n=3)\end{array}$ & Recovery $/ \%$ & $\begin{array}{c}\mathrm{RSD} / \% \\
(n=3)\end{array}$ & Recovery $/ \%$ & $\begin{array}{c}\mathrm{RSD} / \% \\
(n=3)\end{array}$ \\
\hline 3-羟基克百威(Carbofuran-3-hydroxy) & 65 & 7 & 92 & 6 & 85 & 7 \\
\hline 倍硫磷砜(Fenthion-sulfone) & 63 & 14 & 85 & 6 & 78 & 4 \\
\hline 倍硫磷亚砜(Fenthion-sulfoxide) & 84 & 11 & 92 & 4 & 95 & 12 \\
\hline 苯醚甲环唑(Difenoconazole) & 89 & 15 & 113 & 5 & 77 & 3 \\
\hline 吡虫啉(Imidacloprid) & 71 & 8 & 85 & 17 & 78 & 1 \\
\hline 吡䳋酮(Pymetrozine) & 63 & 18 & 73 & 12 & 70 & 13 \\
\hline 残杀威(Propoxur) & 82 & 19 & 93 & 10 & 91 & 12 \\
\hline 虫酰肼(Tebufenozide) & 88 & 13 & 89 & 13 & 91 & 15 \\
\hline 敌稗(Propanil) & 97 & 5 & 113 & 11 & 101 & 10 \\
\hline 敌草胺(Napropamide) & 83 & 8 & 92 & 5 & 89 & 7 \\
\hline 丁草胺(Butachlor) & 73 & 13 & 101 & 10 & 88 & 11 \\
\hline 定菌磷(Pyrazophos) & 100 & 6 & 101 & 15 & 91 & 7 \\
\hline 啶虫脒(Acetamiprid) & 78 & 14 & 84 & 2 & 90 & 4 \\
\hline 对嘧菌环胺(Cyprodinil) & 78 & 7 & 97 & 3 & 88 & 1 \\
\hline 丰索磷(Fensulfothion) & 79 & 14 & 80 & 10 & 84 & 1 \\
\hline 甲草胺(Alachlor) & 66 & 16 & 73 & 5 & 74 & 9 \\
\hline
\end{tabular}


续表

\begin{tabular}{|c|c|c|c|c|c|c|}
\hline \multirow[b]{2}{*}{ Compd. } & \multicolumn{2}{|c|}{ Spiked $10 \mu \mathrm{g} / \mathrm{kg}$} & \multicolumn{2}{|c|}{ Spiked $50 \mu \mathrm{g} / \mathrm{kg}$} & \multicolumn{2}{|c|}{ Spiked $100 \mu \mathrm{g} / \mathrm{kg}$} \\
\hline & Recovery/\% & $\begin{array}{c}\mathrm{RSD} / \% \\
(n=3)\end{array}$ & Recovery/\% & $\begin{array}{c}\mathrm{RSD} / \% \\
(n=3)\end{array}$ & Recovery $/ \%$ & $\begin{array}{c}\mathrm{RSD} / \% \\
(n=3)\end{array}$ \\
\hline 甲慕威(Carbaryl) & 105 & 11 & 96 & 11 & 87 & 11 \\
\hline 甲霜灵(Metalaxyl) & 69 & 14 & 83 & 10 & 77 & 8 \\
\hline 腈菌唑(Myclobutanil) & 95 & 11 & 97 & 8 & 84 & 14 \\
\hline 抗蚜威(Pirimicarb) & 84 & 7 & 78 & 7 & 94 & 2 \\
\hline 克百威(Carbofuran) & 80 & 10 & 87 & 5 & 76 & 19 \\
\hline 喹禾灵(Quizalofop-ethyl) & 89 & 5 & 97 & 8 & 95 & 5 \\
\hline 磷胺(Phosphamidon) & 85 & 12 & 81 & 4 & 104 & 8 \\
\hline 氯唑啉(Isazofos) & 90 & 1 & 98 & 1 & 89 & 7 \\
\hline 咯菌腈(Fludioxonil) & 99 & 6 & 113 & 9 & 99 & 3 \\
\hline 马拉氧磷(Malaoxon) & 81 & 12 & 88 & 4 & 90 & 8 \\
\hline 猛杀威(Promecarb) & 96 & 11 & 75 & 13 & 75 & 13 \\
\hline 嘧啶磷(Pirimiphos-ethyl) & 80 & 7 & 110 & 5 & 97 & 2 \\
\hline 嘧菌酯(Azoxystrobin) & 91 & 9 & 84 & 7 & 85 & 9 \\
\hline 灭多威(Methomyl) & 73 & 13 & 78 & 9 & 75 & 6 \\
\hline 灭菌磷(Ditalimfos) & 102 & 14 & 100 & 12 & 95 & 6 \\
\hline 灭克磷(Ethoprophos) & 89 & 8 & 100 & 5 & 88 & 10 \\
\hline 噻虫嗪(Thiamethoxam) & 78 & 16 & 72 & 8 & 74 & 5 \\
\hline 噻嗪酮(Buprofezin) & 83 & 9 & 97 & 12 & 101 & 7 \\
\hline 杀铃脲(Triflumuron) & 113 & 6 & 106 & 7 & 95 & 12 \\
\hline 三唑醇(Triadimenol) & 107 & 9 & 97 & 14 & 95 & 14 \\
\hline 三唑䣳(Triadimefon) & 85 & 15 & 98 & 11 & 83 & 8 \\
\hline 速灭威(Metolcarb) & 83 & 20 & 93 & 6 & 89 & 6 \\
\hline 特谱唑(Diniconazole) & 88 & 12 & 105 & 4 & 85 & 13 \\
\hline 涕灭威砜(Aldicarb-sulfone) & 100 & 12 & 80 & 9 & 90 & 12 \\
\hline 戊唑醇(Tebuconazole) & 89 & 11 & 106 & 7 & 79 & 2 \\
\hline 西玛津(Simazine) & 101 & 10 & 89 & 9 & 97 & 5 \\
\hline 乙嘧硫磷(Etrimfos) & 76 & 14 & 72 & 3 & 81 & 14 \\
\hline 异丙甲草胺(Metolachlor) & 91 & 10 & 91 & 2 & 83 & 7 \\
\hline 异丙威(Isoprocarb) & 73 & 14 & 76 & 15 & 82 & 11 \\
\hline 仲丁威(Fenobucarb) & 81 & 7 & 87 & 3 & 81 & 2 \\
\hline
\end{tabular}

\section{3 结论}

实验建立了 MSPD-UPLC-MS/MS 法对人参中药材 中的 46 种农药残留进行分析, 考察了方法的线性范围、 线性方程、定量限、加标回收率及 RSD，同时对 MSPD 分散剂、洗脱剂的种类与配比、洗脱剂体积等参数进行 了优化. 实验结果表明, 该方法成本低且简便、快速，具 有良好的适用性与普遍的实用价值.

\section{References}

1 Chen, D.; Chu, L.-W.; Hou, Z.-G.; Shang, M.; Fan, Z.-X.; Li, Y.-R. Chin. J. Appl. Chem. 2007, 24(2), 210 (in Chinese).

(陈丹, 初丽伟, 侯志广, 尚梅, 范志先, 李月茹, 应用化 学, 2007, 24(2), 210.)

2 Nguyen, T. D.; Han, E. M.; Seo, M. S.; Kim, S. R.; Yun, M. Y.; Lee, D. M.; Lee, G.-H. Anal. Chim. Acta 2008, 619, 67.

3 Nguyen, T. D.; Yu, J. E.; Lee, D. M.; Lee, G.-H. Food 
Chem. 2008, 110, 207

4 Fillâtre, Y.; Rondeau, D.; Bonnet, B.; Daguin, A.; Jadas-Hécart, A.; Communal, P. Y. Anal. Chem. 2011, 83, 109.

5 Fenoll, J.; Hell1'n, P.; Martínez, C. M.; Flores, P. Chromatographia 2010, 72, 857. Lee, J. M.; Park, J. W.; Jang, G. C.; Hwang, K. J. J. Chromatogr. A 2008, 1187, 25.

6 Alder, L.; Greulich, K.; Kempe, G.; Vieth, B. Mass Spectrom. Rev. 2006, 25, 838.

7 Tan, J.; Liu, X.-Y.; Zhao, L.-M.; Lu, Y.; Liu, Z.-H. Acta Chim. Sinica 2009, 67(20), 2385 (in Chinese).

(谭君, 刘小叶, 赵柳曼, 陆英, 刘仲华, 化学学报, 2009, 67(20), 2385.)

8 Qin, Q.; Wu, D.; Li, X.-H.; Song, W.-B. J. Food Sci. Bio-
Biotechnol 2007, 26(5), 57 (in Chinese).

(秦琴, 吴迪, 李新华, 宋文斌, 食品与生物技术学报, 2007, 26(5), 57.)

9 Liu, D.-J.; Xue, J.; Cui, C. China J. Chin. Mater. Med 2008, 33(20), 2421 (in Chinese).

(刘东静, 薛健, 崔澈, 中国中药杂志, 2008, 33(20), 2421.)

10 Fillion, J.; Hindle, R.; Lacroix, M.; Selwyn, J. J. AOAC Int. 1995, 78, 1252.

11 Douglas, G. H.; Jon, W. W. Anal. Chem. 2009, 81(14), 5716.

12 Barker, S. A.; Long, A. R.; Short, C. R. J. Chromatogr. A 1989, 475, 353.

13 Dagnac, T.; Garcia-Chao, M.; Pulleiro, P.; Garcia-Jares, C.; Llompart, M. J. Chromatogr. A 2009, 1216, 3702.

(A1110262 Qin, X.) 